\title{
Connectedness and Compactness on Standard Sets
}

\author{
Ricardo Almeida \\ ricardo.almeida@ua.pt \\ Department of Mathematics, University of Aveiro, 3810-193 Aveiro, Portugal
}

\begin{abstract}
We present a nonstandard characterization of connected compact sets

Mathematics Subject Classification 2010: 26E35, 54D05, 54D30.
\end{abstract}

Keywords: Nonstandard analysis, Connected set, Compact sets

\section{Introduction}

There is as yet no simple nonstandard characterization of connectedness, and little work has been done in that direction. In [4], Steven Leth presents a sufficient condition for a set $A \subseteq \mathbb{R}^{n}$ to be connected. As Leth remarked, however, it is not a necessary condition. His hypothesis involves internal polygonal paths joining distinct points. We will work with hyper-finite sets instead of polygonal paths, thus eliminating the implicit local path-connectedness that is present in [4]. We mention also the work of Sérgio Rodrigues [5] characterizing connectedness in nonstandard terms using the monad of a set.

The paper is organized as follows. In section 2 we collect some necessary background for the reader's convenience. In section 3 we present new results about connectedness and compactness on standard sets; we introduce a new concept, the discrete infinitesimal path, which will be used to characterize connected compact sets in metric spaces.

\section{Preliminaries}

All the sets which come up in classical analysis have nonstandard extensions using a map denoted by "*". For example, if $\mathbb{R}$ denotes the set of real numbers, * $\mathbb{R}$ will be its nonstandard extension. This extension contains "ideal elements", like infinitesimals and infinite numbers, but also a copy of the set of real numbers, denoted by the symbol ${ }^{\sigma} \mathbb{R}$. It is not our intention to give a full exposition on this subject, we will just fix notation and present some results needed. For further details, the reader is referred to $[2,3,6,8]$.

Definition 1. Let $x, y \in{ }^{*} \mathbb{R}$. We say that

1. $x$ is infinitesimal if $|x|<\epsilon$, for all positive real number $\epsilon$ and we write $x \approx 0$;

2. $x$ is finite if, for some positive real number $\epsilon,|x|<\epsilon$;

3. $x$ is infinite (or infinitely large) if it is not finite, i.e., for any positive real number $\epsilon,|x|>\epsilon$; we write $x \approx \infty$;

4. $x, y$ are infinitely close if $x-y$ is infinitesimal; we write $x \approx y$.

In the following, $(X, d)$ is a metric space. 
Definition 2. For $x \in{ }^{*} X$, the monad of $x$ is the subset of ${ }^{*} X$ given by

$$
\mu(x):=\left\{y \in^{*} X \mid d(x, y) \approx 0\right\} .
$$

As before, the nonstandard extension of $X$ contains a copy of the original set, which we denote by ${ }^{\sigma} X$ (elements of ${ }^{\sigma} X$ are called standard). A point $y \in{ }^{*} X$ is nearstandard if there exists some standard $x \in{ }^{\sigma} X$ such that $y \in \mu(x)$; in this case we say that $x$ is the standard part of $y$ and write $s t(y)=x$. We say that $x, y \in{ }^{*} X$ are infinitely close, and write $x \approx y$, if $d(x, y) \approx 0$. If $x$ and $y$ are not infinitely close, we write $x \not \approx y$.

The set of the nearstandard points of ${ }^{*} X$ is

$$
n s\left({ }^{*} X\right):=\bigcup\left\{\mu(x) \mid x \in{ }^{\sigma} X\right\} .
$$

Theorem 1. [3] Let $A \subseteq X$. Then

1. $A$ is open if and only if for all $a \in{ }^{\sigma} A, \mu(a) \subseteq{ }^{*} A$ holds;

2. $A$ is closed if and only if, whenever $a \in *^{*} A$ and $a \approx x$ for some $x \in{ }^{\sigma} X$, we have $x \in{ }^{\sigma} A$;

3. $A$ is compact if and only if for all $a \in{ }^{*} A$, there is an $x \in{ }^{\sigma} A$ with $a \approx x$;

In every metric space, monads of distinct standard points are disjoint (see [3]). Therefore, for all $x \in n s\left({ }^{*} X\right)$, there exists exactly one element in ${ }^{\sigma} X$, called $s t(x)$, infinitely close to $x$. Hence we have a well-defined function

$$
\begin{array}{cl}
\text { st }: \quad n s\left({ }^{*} X\right) & \rightarrow{ }^{\sigma} X \\
x & \mapsto s t(x)
\end{array}
$$

called the standard part function.

Theorem 2. [3] Let $X$ and $Y$ be two topological spaces and $f: X \rightarrow Y$ a function. Then $f$ is continuous if and only if

$$
\forall x \in{ }^{\sigma} X \quad f(\mu(x)) \subseteq \mu(f(x)),
$$

or equivalently,

$$
\forall x \in{ }^{\sigma} X \forall y \in{ }^{*} X \quad[x \approx y \Rightarrow f(x) \approx f(y)] .
$$

\section{Main results}

In what follows, $\left(X, d_{X}\right)$ and $\left(Y, d_{Y}\right)$ will denote two metric spaces, and $A \subseteq X$ a nonempty subset. To simplify notation, we will denote both metrics by the same symbol $d$. Given two points $x, y \in{ }^{*} A$, we define the set (possibly external)

$$
\begin{aligned}
\mathcal{P}_{x, y}^{*} A= & \left\{u=\left(u_{n}\right)_{n=1, \ldots, N} \mid N \in{ }^{*} \mathbb{N}, u_{1}=x, u_{N}=y, u_{n} \in{ }^{*} A\right. \\
& \text { and } \left.u_{n} \approx u_{n+1}, \text { for all } n \in\{1, \ldots, N-1\}\right\} .
\end{aligned}
$$

We call the hyper-finite sequence $u=\left(u_{n}\right)_{n \in\{1, \ldots N\}}$ a discrete infinitesimal path (abbreviation d.i.p.) joining $x$ to $y$ in ${ }^{*} A$. We define a binary relation on ${ }^{*} A$ by $x \sim y$ if $\mathcal{P}_{x, y}^{*}$ is nonempty; it is easy to prove that $\sim$ is an equivalence relation.

We will simply write $\mathcal{P}_{x, y}$ instead of $\mathcal{P}_{x, y}^{* A}$ whenever there is no danger of confusion.

The existence of (standard) discrete paths joining points on connected sets is known. In fact, it can be proved that if $A$ is a connected set and $\epsilon$ is a (standard) real, then for all $x, y \in A$, there exists a finite sequence of points, all lying in $A$,

$$
x=u_{1}, u_{2}, \ldots, u_{n}=y,
$$

such that the distance between any two successive points in this sequence is less than $\epsilon$. $f$.

The next result follows from the compactness of $A$ and the consequent uniform continuity of 
Theorem 3. Let $f: X \rightarrow Y$ be a function. If $f$ is continuous, then for any subset $A \subseteq X$ satisfying

$$
\forall x, y \in{ }^{*} A \exists u \in \mathcal{P}_{x, y} \text { with } u_{n} \in n s\left({ }^{*} X\right) \text { and } \operatorname{st}\left(u_{n}\right) \in{ }^{\sigma} A, \text { for all } n
$$

the following condition is valid

$$
\forall z, w \in{ }^{*} f(A) \exists v \in \mathcal{P}_{z, w} \text { with } v_{n} \in n s\left({ }^{*} Y\right) \text { and st }\left(v_{n}\right) \in{ }^{\sigma} f(A) \text {, for all } n .
$$

Proof. Let $A$ be a set that satisfies condition (1). Given $z$ and $w$ in ${ }^{*} f(A)$, let $z=f(x)$ and $w=f(y)$, for some $x, y \in{ }^{*} A$. Then, there exists $u=\left(u_{n}\right)_{n=1, \ldots, N} \in \mathcal{P}_{x, y}$, such that $u_{n} \in n s\left(^{*} X\right)$ and $\operatorname{st}\left(u_{n}\right) \in{ }^{\sigma} A$, for all $n=1, \ldots, N$. Define $v_{n}:=f\left(u_{n}\right)$, for all $n=1, \ldots, N$. It is easy to see that $v=\left(v_{n}\right)$ satisfies the necessary conditions.

Theorem 4. The set $A$ is connected if

$$
\forall x, y \in{ }^{\sigma} A \exists u \in \mathcal{P}_{x, y} \text { with } u_{n} \in n s\left({ }^{*} X\right) \text { and } \operatorname{st}\left(u_{n}\right) \in{ }^{\sigma} A \text {, for all } n \text {. }
$$

Proof. Assume that $A$ is not connected. Then $A$ has a subset $B \notin\{\emptyset, A\}$ that is simultaneously relatively open and closed. Pick $x \in{ }^{\sigma} B, y \in{ }^{\sigma}(A-B)$ and $u=\left(u_{n}\right)_{n=1, \ldots, N} \in \mathcal{P}_{x, y}$ such that $u_{n} \in n s\left({ }^{*} X\right)$ and $s t\left(u_{n}\right) \in{ }^{\sigma} A$, for all $n$, and define the internal set

$$
K:=\left\{n \in\{1, \ldots, N\} \mid u_{n} \in{ }^{*} B\right\} .
$$

Since $K$ is nonempty (for example, $1 \in K$ ), it has a maximum. Let $k:=\max K$. Since $y \notin{ }^{*} B$ then $k \neq N$. Besides this, $u_{k} \in{ }^{*} B$ and $u_{k+1} \in{ }^{*}(A-B)$. Since $B$ and $A-B$ are both closed, $s t\left(u_{k}\right) \in B$ and $s t\left(u_{k+1}\right) \in A-B$.

Since $u_{k} \approx u_{k+1}$, the point $\operatorname{st}\left(u_{k}\right)=\operatorname{st}\left(u_{k+1}\right) \in{ }^{\sigma} B \cap^{\sigma}(A-B)$, which ends the proof.

The previous condition is not enough to assert that $A$ is path connected; e.g. take the set

$$
\{(x, \sin (1 / x)) \mid x>0\} \cup(\{0\} \times[-1,1]) .
$$

However, if $A$ is path connected then condition (2) is satisfied. Indeed, if we fix $x, y \in{ }^{\sigma} A$, then by hypothesis there exists a continuous path $\alpha:[0,1] \rightarrow A$ with $\alpha(0)=x$ and $\alpha(1)=y$. Take an infinite $N \in{ }^{*} \mathbb{N}$ and define $u_{n}:=\alpha\left(\frac{n}{N}\right)$ for $n \in\{0, \ldots, N\}$. It is easy to check that $\left(u_{n}\right)$ satisfies condition (2).

The converse of Theorem 4 is false in general, however we will obtain a related result.

Theorem 5. If $A$ is a connected set then for all $x, y \in{ }^{*} A$ the condition $\mathcal{P}_{x, y} \neq \emptyset$ holds.

Proof. Fix $x, y \in{ }^{\sigma} A$ and $\epsilon \in{ }^{\sigma} \mathbb{R}^{+}$. Then there exists an $\epsilon$-chain that joins $x$ and $y$ (c.f. [7], pag 120). Therefore

$$
\begin{gathered}
\forall x, y \in{ }^{\sigma} A \forall \epsilon \in{ }^{\sigma} \mathbb{R}^{+} \exists N \in{ }^{\sigma} \mathbb{N} \exists\left\{u_{2}, \ldots, u_{N-1}\right\} \subset{ }^{\sigma} A \\
\forall i \in\{1, \ldots, N-1\} \quad d\left(u_{i}, u_{i+1}\right)<\epsilon,
\end{gathered}
$$

where $u_{1}:=x$ and $u_{N}:=y$. Now, pick two points $x, y \in{ }^{*} A$. By the Transfer Principle, condition holds with $\epsilon \approx 0$.

Observe that we actually proved that, for all infinitesimal $\epsilon$, there exists $u \in \mathcal{P}_{x, y}$ satisfying $d\left(u_{i}, u_{i+1}\right)<\epsilon$.

Unfortunately, the d.i.p. need not to be nearstandard in $A$, as is shown in the next example.

Let $A$ be the subset of $\mathbb{R}^{2}$ defined by

$$
([0,1] \times\{0\}) \cup\left\{\left(\frac{1}{n}, y\right) \mid n \in \mathbb{N}, y \in[0,1]\right\} \cup\{(0,0),(0,1)\} .
$$

The set $A$ is connected but there is no d.i.p. joining the points $(0,0)$ to $(0,1)$ nearstandard in the set. 
Corollary 1. Let $A$ be a compact set. Then $A$ is connected if and only if

$$
\forall x, y \in{ }^{\sigma} A \exists u \in \mathcal{P}_{x, y} \text { such that } u_{n} \in n s\left({ }^{*} X\right) \text { and } s t\left(u_{n}\right) \in{ }^{\sigma} A \text {, for all } n .
$$

Proof. Follows from Theorems 4 and 5 and the fact that, for the nonstandard extension of compact sets, all points are nearstandard on the set.

In conclusion, we have now a nice characterization of connected compact sets.

Corollary 2. Let $A$ be a non-empty set. Then $A$ is connected and compact if and only if

$$
\forall x, y \in{ }^{*} A \exists u \in \mathcal{P}_{x, y} \text { such that } u_{n} \in n s\left({ }^{*} X\right) \text { and } \operatorname{st}\left(u_{n}\right) \in{ }^{\sigma} A \text {, for all } n .
$$

Proof. We only need to prove that condition (3) implies the compactness condition. Fix $x \in{ }^{*} A$. By condition (3), there exists some $u \in \mathcal{P}_{x, x}$ nearstandard on $A$. So $u_{1}=x \in n s\left({ }^{*} X\right)$ and $\operatorname{st}(x) \in{ }^{\sigma} A$.

\section{Acknowledgments}

Work supported by Centre for Research on Optimization and Control (CEOC) from the "Fundação para a Ciência e a Tecnologia" (FCT), cofinanced by the European Community Fund FEDER/POCI 2010 .

\section{References}

[1] R. Almeida, On the continuity of functions, Appl. Sci. (9), pp 1-4 (2007).

[2] R. Almeida and D.F.M. Torres, Relaxed optimality conditions for mu-differentiable functions, Int. J. Appl. Math. \& Stat. (14), No. M09 pp 53-66 (2009).

[3] A.E. Hurd and P.A. Loeb, An Introduction to Nonstandard Real Analysis, Pure and Applied Mathematics, 118. Orlando etc., Academic Press, Inc. (1995).

[4] S.C. Leth, Some Nonstandard Methods in Geometric Topology, in N.J. Cutland, V. Neves, F. Oliveira and J. Sousa-Pinto (Eds.), Developments in Nonstandard Mathematics, Pitman Research Notes in Mathematics Series. 336, New York (1995).

[5] S. Rodrigues, Propriedades da Separação, Conexão e Real-compacidade, Master's thesis, University of Aveiro (2001).

[6] A. Robinson, Non-Standard Analysis, North- Holland Publishing Company, Amsterdam (1974).

[7] W. Sierpinski, General topology, (translated by C. Krieger), Dover Publications, Inc., Mineola, New York, (2000).

[8] K. D. Stroyan and W. A. J. Luxemburg, Introduction to the theory of Infinitesimals, Pure Appl. Math. 72 New York-San Francisco-London, Academic Press (1976). 